\title{
Color vision screening without the use of technical equipment: Scale development and cross-validation
}

\author{
STANLEY COREN and A. RALPH HAKSTIAN \\ University of British Columbia, Vancouver, British Columbia, Canada
}

\begin{abstract}
Large-scale normative samples of data dealing with visual function and their relationships to individual difference variables are relatively scarce. This scarcity is, in part, due to the fact that data collection usually requires individual testing and the use of technical equipment and trained personnel rather than simple survey instruments. Using a sample of 175 individuals, we developed a self-report behavioral inventory, suitable for group testing or survey administration, to assess color vision, and validated it against laboratory tests. The inventory was then cross-validated in a separate sample of 596 subjects. The resultant brief 10 -item scale was shown to be a valid and reliable predictor of color-vision deficit. Hit- and miss-rate tables were developed, and these are presented to allow investigators to select classification criteria appropriate for their purposes. A copy of the inventory and scoring procedure is appended to this report.
\end{abstract}

There are many individual-difference variables that affect visual function. For instance, within normal populations, it is well established that there are systematic changes in visual acuity, color vision, threshold sensitivity, and other functions as a consequence of aging (see Coren, Porac, \& Ward, 1984, Corso, 1981, and Sekuler, Kline, \& Dismukes, 1982, for reviews). In addition, specific visual functions, such as color vision, may be modified by environmental factors, exposure to pharmacological agents, hormonal factors, or specific disease states (e.g., Lakowski, Aspinall, \& Kinnear, 1973; Pokorny, Smith, Verriest, \& Pinckers, 1979). Our understanding of the nature and prevalence of these individual-difference factors in vision, however, is hampered by the fact that normative data, based upon large-scale surveys of a general population, are relatively scarce when it comes to visual function. Although the sparseness of the available data may be due to the small number of perceptual researchers trained in epidemiological and survey techniques, it is more apt to be due to the difficulties involved in obtaining measures of visual function from large, representative samples of individuals. The optimal method of obtaining data about visual capacity is, of course, direct laboratory or clinical measurement. Here one encounters a series of technical, staffing, and cost barriers. The technical problems arise because of the need to have specialized equipment and testing conditions. Even something as apparently simple as testing for color vision, for in-

This work was supported, in part, by grants from the Natural Sciences and Engineering Research Council of Canada, the Koerner Foundation, and the British Columbia Health Care Research Foundation. We would like to acknowledge the contributions of Wayne Wong, Joan Donelly, and Lynda Berger, who assisted in data collection. Requests for reprints should be sent to Stanley Coren, Department of Psychology, University of British Columbia, Vancouver, BC V6T IW5, Canada. stance, requires special test stimuli and specified light sources. If one wishes data on other aspects of visual function (e.g., stereopsis, dark adaptation, acuity, or visualfield integrity), the amount of equipment required increases rapidly and its portability begins to decrease. Increases in the technical level of equimentation inevitably lead to increases in the need for trained personnel to administer the tests and to interpret the data. Such personnel are often expensive or not available.

A striking example of how technical and staffing requirements can hamper acquisition of visual data comes from the Health and Nutrition Examination Survey (HANES) conducted by the U.S. National Eye Institute (Miller, 1976). The vision examinations, of course, required individuals to come to a testing center at which the equipment was available. Because of this inconvenience and the amount of time involved, and despite much encouragement, nearly $30 \%$ of the probability sample failed to come in for their examinations. Staffing problems, namely difficulty in recruiting examiners, ultimately terminated the project in 1972. Ederer (1983) has suggested that the task of conducting numerous examinations, day after day, of subjects who fell mostly in the normal, disease-free range was an inadequate challenge to hold the interest of the ophthalmologists. The alternative to general population surveys has been the collection of visual-status data from large, relatively "static" or "captive"' samples, such as from schools, hospitals, prisons, or military settings, where sensory screening equipment and trained personnel are more readily available (e.g., Meyers, 1981; Pearce, Reed, \& Hofstetter, 1972; Sorsby, Sheridan, \& Leary, 1960). Unfortunately, the selectivity inherent in such an approach severely limits the generalizability of the obtained results, and prevents the emergence of a valid picture of the distribution of visual function in the population at large. 
The difficulties encountered in the assessment of visual status are, of course, not unique. In many other areas, similar problems have been encountered. For instance, the need for relatively inexpensive health-status inventories that can be administered to large populations by nontechnically trained individuals has led to the development of a number of self-report inventories for specific survey purposes. Over the past 2 decades, a number of such inventories, oriented toward specific disease states, have been tested and validated. Several inventories of general health status have also achieved a degree of success. These have been shown to correlate quite well with the clinical judgments of medical practitioners, thus demonstrating their validity (e.g., Bergner, Bobbitt, Carter, \& Gilson, 1981; Maddox \& Douglas, 1964; Parkerson et al., 1981). Unfortunately, similar objectively scored inventories to be used in assessing visual function have not yet been developed.

To date, survey instruments that assess visual function via self-report inventories have involved only global, unvalidated questionnaires. One example is the U.S. Public Health Service Survey 1960-1963, which asks for a self-rating of visual acuity on a 5-point scale ranging from normal vision to blind (Schein, Gentile, \& Haase, 1970). No attempt at validation is offered for this scale. Unfortunately, there is evidence that such global self-reports of visual capacity often produce unreliable and erroneous data. Many people suffer from severe sensory problems, yet are not aware that they are any different from the general population. Coren and Porac (1975), for example, demonstrated that among a sample of adults who confidently believed that they had normal vision, $13.5 \%$ actually had visual acuities that fell in the range of 20/40 or below (the accepted cutoff for the legal definition of impaired vision in many states in the U.S.). Similarly, it is not unusual for individuals to learn that they are color deficient, have poor acuity, or lack depth perception only when they are medically tested as adults, when, for example, they apply for positions in the military or large organizations where routine sensory screening may be required as part of the application process (see Newcomb, 1976).

Although global self-reports of function may be suspect, there are principles involved that may provide a basis upon which to construct valid self-report-based screening inventories that could, potentially, measure particular aspects of visual function. The general solution used in other contexts involves the use of specific items that are behaviorally anchored (e.g., Bennett \& Ritchie, 1975; Rosser, 1983). Use of self-reports based upon responses to frequently encountered situations has led to the development of some reasonably successful health inventories (e.g., Bergner et al., 1981; Parkerson et al., 1981; Wilcock, 1979). Using such a procedure with a modest-sized sample of individuals, Coren and Hakstian (1987) were recently able to provide preliminary data suggesting the feasibility of developing objectively validated scales to measure several different aspects of visual function. Thus, although individuals might not know whether their color vision falls within the normal range, they could certainly answer such specific questions as "Do you have difficulty discriminating between brown and green?" with a reasonable degree of accuracy. A score based upon the composite of a number of such responses on such a scale might well provide an accurate estimate of an individual's color vision. This report describes an attempt to develop and validate such a scale for assessing the status of an individual's color vision.

For such a color-vision screening inventory to have value as a research instrument, it must meet several objectives. Specifically, it must be: (1) valid, as tested against standard laboratory and clinical tests for color deficiency; (2) statistically reliable; (3) based upon information reported by the person being evaluated; (4) suitable for group or postal administration; (5) capable of measuring the broad range of sensory function, from normal through deficient; (6) capable of measuring visual function independently of specific disease states; (7) applicable across all adult age groups (e.g., 18 years and older); (8) brief and easy to comprehend; (9) suitable for selfadministration (or for oral administration by nontechnically trained personnel, should literacy or sensory capacity be very deficient); (10) easily reproducible in format, requiring no special color plates or pictorial matter; (11) able to produce results that may be meaningfully interpreted in terms of the presence or absence of a colorvision deficiency; and (12) concerned with information that is not dependent upon previous clinical diagnoses or direct knowledge of previous color-vision testing.

The following report describes two studies undertaken in the development of a color-vision screening inventory that meets these criteria. The first is the initial developmental study in which the items were behaviorally validated against actual laboratory measures. The second study involved a cross-validation of the inventory using a larger sample.

\section{EXPERIMENT 1: DEVELOPMENT OF THE INVENTORY}

\section{Methods}

We considered it important that the survey instrument being developed not simply reflect the subject's knowledge of the results of any previous color-vision testing based on more traditional laboratory techniques that may have been conducted in the course of medical treatment or other forms of assessment. Our aim was to develop a means by which level of visual function could be assessed solely through behaviorally based self-reports. For this reason, individuals who reported that they had ever failed a color-vision test were excluded from the development sample. Although this restriction of the subject population might be expected to cause an apparent decrease in discriminability of the resultant instrument, we considered it important that responses be based upon direct self-assessment and not upon "second-hand" test reports. To maximize the likelihood that an adequate sample of color-weak individuals would be found, the initial sample was restricted to males, who are approximately 16 times more likely to show a color-vision deficit than are females. A random sample of 175 male university students, 
with a mean age of 18.6 years, was employed. All subjects were naive as to the purpose of the experiment.

The subjects were tested individually. Testing took place in two sessions, separated by at least 1 day. In the first session, a set of 96 color-related self-report items were administered, embedded in a large, 357-item, general inventory of visual function. The color items were based upon commonly reported confusions among colordeficient individuals, extracted from case reports, perceptual assays, and symptom lists. Each of the items was presented as a question with five response categories-Never, Seldom, Occasionally, Frequently, and Always.

The second session was used to obtain an objective assessment of color vision. The separation of testing into two sessions and the order of these sessions were designed to minimize potential confounding between self-reports and knowledge of performance on the objective measure of color vision. Color vision was assessed using the Hardy Rand Rittler (HRR) pseudo-isochromatic plates, administered under a correlated source $C$ illuminant with an intensity of $310 \mathrm{~lx}$. These plates are of the vanishing type, and the hues in the symbols to be identified in the screening series are close to gray. If a subject made an error on any of the six screening plates, the set of plates was inverted and the sequence was repeated in reverse order. If, on retest, the subject still made an error on one or more of the plates, he was classified as color-defective. Used in this manner as a dichotomous color-screening test, HRR plates have been shown to have validity coefficients ranging between 0.88 and 0.96 in tests against an anomaloscope (see Pokorny et al., 1981, for a full review), and thus provide a good, stable laboratory measure of color-vision status.

\section{Results and Discussion}

In this all-male sample, $8.6 \%$ proved to be color weak, as measured by the HRR plates; this is a close approximation of the usually obtained population rate of color deficiency in males. This is probably not surprising inasmuch as $\mathbf{9 7 . 7 \%}$ of the population tested reported that they had no memory of ever being tested for color vision before. Point-biserial correlations between the dichotomous classification of color vision and the responses to each individual item were then computed. The items with the highest correlations were retained. After elimination of redundant items and of items that might be inappropriate for use on a broader cross-section of the general population than was used here, the resultant scale contained 10 items. This scale, now called the Color Vision Screening Inventory (or CSI) is presented in the appendix to this report.

The reliability and validity of this short scale were assessed as follows:

Reliability (internal consistency) was assessed by computation of Cronbach's (1951) alpha coefficient. The obtained alpha was 0.91 , showing high internal consistency among the items.

The predictive validity of the scale was assessed by relating the total (or composite) CSI scale scores to the dichotomous outcome criterion based on the objective assessment of color vision obtained using the HRR plates. The individual-item scores, ranging from 1 (for Never) to 5 (Always), were summed first to give a total CSI scale score. Thus, in this composite scale score, higher scores corresponded to self-ratings of poorer color vision. Individuals were then classified as "normal" or "color weak" on the basis of their objective scores on the HRR test using the criterion indicated above. Table 1 contains the results from this developmental sample. The reader can easily assess, from this table, the degree of correct classification obtained using various CSI total scores as cutoff values. The far-left column contains a range of CSI scores that could be used as cutoff values. The next column, labeled "Normals," contains the percentage of the sample that was classified as normal by the HRR test who obtained a CSI score less than the indicated total. The following column, labeled "Color Weak," contains the percentage of the sample classified as color deficient by the HRR test who obtained a CSI scale value greater than or equal to the indicated value. Thus, these two columns indicate the percentages of each group that are correctly classified if one selects any given total CSI score as a cutoff point. The final column of Table 1 indicates the percentage of the total population correctly classified (the "overall hit rate") by using that particular value as a cutoff point.

Table 1 suggests that the self-report color scale does a reasonable job of correctly classifying individuals on the basis of color vision. For example, $88.9 \%$ of color normals have a CSI score of less than 17 , while $93.3 \%$ of the color-deficient group have scores greater than or equal to this value, resulting in correct classification of $89.1 \%$ of the total sample of 175 subjects. To assess the statistical significance of these classifications, $2 \times 2$ chisquare tests were conducted for each of the cutoff points. As indicated in Table 1, all of the cutoff points produced significant chi-square values, with $p<.001$, except for the cutoff of 11 , which produced a significant chi-square but only at the $p<.05$ level. These inferential tests demonstrate the validity of the CSI scale, and suggest that it may be a useful method for rapidly assessing color vision.

Table 1

Percentage of Developmental Sample $(N=175)$ Correctly Classified Against Screening With Pseudo-Isochromatic Plates

\begin{tabular}{cccc}
\hline $\begin{array}{c}\text { Color } \\
\text { Scale }\end{array}$ & $\begin{array}{c}\text { Normals } \\
\text { (Less than) }\end{array}$ & $\begin{array}{c}\text { Color Weak } \\
\text { (Greater than } \\
\text { or equal to })\end{array}$ & $\begin{array}{c}\text { Total } \\
\text { Sample }\end{array}$ \\
\hline 11 & 21.9 & $100.0^{*}$ & 28.6 \\
12 & 43.8 & $100.0 \dagger$ & 48.6 \\
13 & 64.4 & $100.0 \dagger$ & 67.4 \\
14 & 69.4 & $93.3 \dagger$ & 71.4 \\
15 & 80.0 & $93.3 \dagger$ & 81.1 \\
16 & 85.0 & $93.3 \dagger$ & 85.7 \\
17 & 88.9 & $93.3 \dagger$ & 89.1 \\
18 & 93.1 & $80.0 \dagger$ & 92.0 \\
19 & 95.6 & $73.3 \dagger$ & 93.7 \\
20 & 95.6 & $73.3 \dagger$ & 93.7 \\
21 & 97.5 & $73.3 \dagger$ & 95.4 \\
22 & 98.1 & $73.3 \dagger$ & 96.0 \\
23 & 98.8 & $73.3 \dagger$ & 96.6 \\
24 & 99.4 & $60.0 \dagger$ & 96.0 \\
25 & 100.0 & $60.0 \dagger$ & 96.6 \\
\hline
\end{tabular}

Note-Individuals with scores less than the Color Scale Total are classified as "normal" and those with scores greater than or equal to the Color Scale Total are classified as "color weak." Chi-square significant at ${ }^{*} p<.05$ and $\nmid p<.001$. 
Before the CSI can be accepted as a potentially useful means of surveying color vision, it is essential to recognize that the procedures employed here tend to capitalize on chance, since we began by first selecting the questionnaire items that individually predicted the objective measure of color vision and then created a scale consisting of those items. In Experiment 1, we demonstrated the predictive validity of this composite scale using the same sample that was used to select the individual items. To assess its validity when applied to an independent sample of subjects, the scale would have to be cross-validated. It is necessary to do this in order to control for the capitalization upon chance relationships between individual items and the objective criterion that may have occurred in the development sample. Furthermore, since the initial sample was limited to males, it would be important to show that this inventory was generalizable across genders. To effect such a cross-validation, we conducted Experiment 2.

\section{EXPERIMENT 2: CROSS-VALIDATION OF THE COLOR SCALE}

\section{Materials and Methods}

The procedures used in the cross-validation were very similar to those used in the original development study. Two separate sessions were employed (the first for self-report and the second for color-vision testing). In this cross-validation, we did not use a large inventory of items. In the first session, subjects were asked to fill out the 10-item CSI embedded in a much shortened questionnaire on visual status that contained only 48 items (rather than the original 357 items of the development phase). Objective HRR testing and dichotomization of subjects on the color vision criterion were identical to those of the development study. The subject sample was demographically similar to that in the development sample, and the same selection criteria were employed, except that both males and females were tested. With 596 university students (267 male and 329 female), the size of the cross-validation sample, however, was considerably larger. These subjects had a mean age of 18.6 , they were naive as to the purpose of the experiment, and they had no prior diagnosis of color-vision deficit.

\section{Results and Discussion}

The HRR testing showed that $7.1 \%$ of the males and $0.6 \%$ of the females had color-vision deficits, again well within usually accepted ranges. The statistical analyses followed a pattern similar to that used in the development of the CSI, except that the item-selection phase was not needed. The reliability of the scale was again assessed via Cronbach's alpha coefficient. The obtained value of 0.87 for the cross-validation sample was nearly as high as that obtained for the original development sample.

Predictive validity was assessed in a manner similar to that of Experiment 1. Total CSI scores were related to objective color-vision status in the dichotomous form used earlier. This relationship is presented in tabular form in Table 2, in which the classification accuracy as a function of each cutoff score is given in the format used in Table 1.

The overall pattern apparent in Table 2 again attests to the validity of the CSI scores, in terms of their ability to
Table 2

Percentage of Cross-Validation Sample $(N=596)$ Correctly Classified Against Screening With Pseudo-Isochromatic Plates

\begin{tabular}{cccc}
$\begin{array}{c}\text { Color } \\
\text { Scale } \\
\text { Total }\end{array}$ & $\begin{array}{c}\text { Normals } \\
\text { (Less than) }\end{array}$ & $\begin{array}{c}\text { Color Weak } \\
\text { (Greater than } \\
\text { or equal to })\end{array}$ & $\begin{array}{c}\text { Total } \\
\text { Sample }\end{array}$ \\
\hline 11 & 21.6 & 90.5 & 24.0 \\
12 & 40.9 & $90.5 *$ & 42.6 \\
13 & 56.5 & $90.5 \dagger$ & 57.7 \\
14 & 67.3 & $85.7 \dagger$ & 68.0 \\
15 & 77.4 & $85.7 \dagger$ & 77.7 \\
16 & 83.7 & $81.0 \dagger$ & 83.6 \\
17 & 89.0 & $81.0 \dagger$ & 88.8 \\
18 & 92.7 & $71.4 \dagger$ & 91.9 \\
19 & 94.8 & $61.9 \dagger$ & 93.6 \\
20 & 96.0 & $61.9 \dagger$ & 94.8 \\
21 & 97.4 & $61.9 \dagger$ & 96.1 \\
22 & 98.4 & $61.9 \dagger$ & 97.1 \\
23 & 99.0 & $61.9 \dagger$ & 97.7 \\
24 & 99.3 & $52.4 \dagger$ & 97.7 \\
25 & 99.7 & $47.6 \dagger$ & 97.8 \\
\hline
\end{tabular}

Note-Individuals with scores less than the Color Scale Total are classified as "normal" and those with scores greater than or equal to the Color Scale Total are classified as "color weak." Chi-square significant at ${ }^{*} p<.05$ and $\dagger p<.001$.

classify individuals correctly on the basis of objective color-vision tests. For example, $89.0 \%$ of the colornormal individuals had a CSI score of less than 17, while $81.0 \%$ of the color-deficient individuals had a CSI score greater than or equal to 17 . As before, by conducting chisquare tests on the resultant $2 \times 2$ tables for each cutoff point, we found that for every CSI cutoff score selected larger than 12 , the resulting chi-square statistic was significant at the $p<.001$ level. With 12 used as the cutoff point, the chi-square statistic was significant at the $p<.05$ level. These results are particularly impressive when one recalls that the sample deliberately screened out individuals who had prior knowledge of a color-vision defect based upon objective clinical testing.

The results of this cross-validation, therefore, indicate that the Color Vision Screening Inventory is both a valid and a reliable indicator of color-vision deficit.

\section{GENERAL DISCUSSION}

The two studies presented in this report trace the development of a brief, simple, behaviorally validated screening instrument that can classify individuals on the basis of their color-vision status. From a methodological viewpoint, these results are quite promising. The CSI is brief, taking only a few moments to fill out and to score. It could easily be administered by mail. It could be administered by itself (since it appears to work well even in the absence of the distractor items; see below) or as a brief color-vision screen in larger questionnaires, thereby reducing the task demands placed on respondents. No visual apparatus of any kind and no particular lighting or other testing-session requirements are needed. This makes the CSI very attractive for studies in which the assessment of color-vision status of large samples of indi- 
viduals is desirable, either for normative purposes or for subject selection for further studies of color function.

Perhaps an informal example of the use of the CSI might demonstrate these advantages more clearly. One colleague has been doing research on color-vision deficits for many years. His usual procedure is to screen his introductory psychology class for color-vision deficits, using pseudoisochromatic plates to isolate color-weak individuals who might be invited back for further testing. He schedules students for a visit to his laboratory at 10 -min intervals for screening. Laboratory administration is required because an approximate source $\mathrm{C}$ light source is needed for accurate assessment. As a substitute for his usual procedure, we provided him with a "stripped down" version of the CSI (only the 10 color items with no distractors). He then administered this scale to a class of 191 students. Since he was interested in selection rather than description, he utilized a cut point of 18 or greater as designating color-deficients and one of 12 or less as designating color-normals. Using these criteria, 6 presumably colorweak males were isolated and a presumably normal sample, matched for sex, age, and race, was also selected. All 12 individuals were later tested in the laboratory via the HRR plates, and all of the presumably color-weak individuals failed at least one plate, whereas none of the CSI-designated normals did. The probability of this degree of accuracy on the basis of chance alone is less than .0001 (from a binomial test with a nominal population split of 0.9 color-normal and 0.1 color-weak). The total test administration time for the sample of 191 was approximately $10 \mathrm{~min}$; hand-scoring took approximately $2 \mathrm{~h}$. This should be contrasted to the approximately $32 \mathrm{~h}$ of testing time that the usual screening would have taken.

For the perceptual researcher who wishes to employ the CSI, Table 2 provides a useful means for selecting an appropriate cutoff point. Although we might suggest a cutoff value of 17 as providing reasonable balance, researchers can select a cutoff score to fit their own needs. Those interested in capturing more of the color-deficient population and less worried about false positives might select a lower cutoff. For example, in the selection example described above, the cutoff points were selected to minimize erroneous classification. In any event, the tabled percentages allow a clear indication of classification accuracy for any given range of CSI scores. An alternative approach is to use CSI scores as a continuous variable in correlational applications or in studies comparing color-vision status in selected populations or subgroups.

In summary, the Color Vision Screening Inventory appears to represent a reliable and valid means of rapidly determining whether an individual is color-deficient, independently of prior knowledge based upon clinical or laboratory testing. It requires no technical equipment or expertise to administer and may be scored quickly and interpreted easily. Although it is not designed to replace standard individual testing when more precise diagnostic information is needed, this inventory does provide a valid measure of color-vision status, a measure suitable for survey or normative use or for preliminary screening as a precursor to more extensive assessment, if indicated. Since no technical equipment is needed and individual testing is not required, the inventory can be used for group testing or postal surveys of selected populations in which changes in color vision may be related to other individualdifference factors, such as age, familial history, use of pharmacological agents, drugs, tobacco, and so forth. It also suggests that it may be possible to develop self-report inventories that may assess other aspects of visual function as well.

\section{REFERENCES}

Bennett, A. E., \& Ritchie, K. (1975). Questionnaires in medicine. London: Nuffield Provincial Hospitals Trust.

Bergner, M., Bobbitt, R. A., CARTer, W. B., \& Gilson, B. S. (1981). The Sickness Impact Profile: Development and final revision of a health status measure. Medical Care, 19, 787-805.

CoRen, S., \& HAKstian, A. R. (1987). Visual screening without the use of technical equipment: Preliminary development of a behaviorally validated questionnaire. Applied Optics, 26, 1468-1472.

Coren, S., \& Porac, C. (1975). The myth of the normal eye: A methodological note. Bulletin of the Psychonomic Society, 5, 469-470.

COREN, S., PORAC, C., \& WARD, L. M. (1984). Sensation and perception (2nd ed.). Orlando, FL: Academic Press.

Corso, J. F. (1981). Aging sensory systems and perception. New York: Praeger.

Cronbach, L. J. (1951). Coefficient alpha and the internal structure of tests. Psychometrika, 16, 297-334.

EDERER, F. (1983). Methodological problems in eye disease epidemiology. Epidemiologic Reviews, 5, 51-66.

Lakowski, R., Aspinall, P. A., \& Kinnear, P. R. (1973). Association between colour vision losses and diabetes mellitus. Ophthalmological Research, 4, 145-159.

Maddox, G. L. , \& Douglas, E. B. (1964). Self-assessment of health: A longitudinal study of elderly subjects. Journal of Health \& Social Behavior, 14, 87-93.

Meyers, K. J. (1981). Visual acuity of the hospitalized veteran. American Journal of Optometry \& Physiological Optics, 58, 1077-1091.

Miller, H. W. (1976). Plan and operation of the Health and Nutrition Examination Survey, United States 1971-1973. National Center for Health Statistics (Vital \& Health Statistics, Series 1, No. 10a; DHEW Publication No. HRA 76-1370). Washington, DC: U.S. Government Printing Office.

Newcomb, R. D. (1976). Prevalence of undiagnosed eye/vision disorders in a VA hospital. Journal of the American Optometric Association, 47, 1145-1150.

Parkerson, G. R., Gehlbach, S. H., Wagner, E. H., James, S. A., Clapp, N. E., \& Muhlbaier, L. H. (1981). The Duke-UNC Health Profile: An adult health status instrument for primary care. Medical Care, 19, 806-828.

Pearce, R. L., Reed, D. R., \& Hofstetter, H. W. (1972). Optometric data characteristics of male prison inmates. American Journal of Optometry \& Archives of Physiological Optics, 49, 661-672.

Pokorny, J., Collins, W., HowetT, G., Lakowski, R., Lewis, M., Moreland, J., Paulson, H., \& Smith, V. C. (1981). Procedures for color vision testing: Report of working group 41. Washington, DC: National Academy Press.

Pokorny, J., Smith, V. C., Verriest, G., \& Pinckers, A. J. L. G. (1979). Congenital and acquired color vision defects. New York: Grune \& Stratton.

Rosser, R. (1983). Issues of measurement in the design of health indicators: A review. In A. J. Culyer (Ed.), Health indicators (pp. 3481). Oxford: Martin Robertson. 
Schein, J. D., Gentil.e, A., \& HaAse, K. W. (1970). Development and evaluation of an expanded hearing loss scale questionnaire (National Center for Health Statistics, Series 2, No. 37). Rockville, MD: U.S. Dept. of Health, Education \& Welfare.

Sekuler, R., Kline, D., \& Dismukes, K. (1982). Aging and human visual function. New York: Alan R. Liss.
Sorsby, A., Sheridan, M. \& Leary, G. (1960). Vision, visual acuity and ocular refraction of young men: Findings in a sample of 1,033 subjects. British Medical Journal, 1, 1394-1398.

WiLcock, G. K. (1979). Use of a self-administered postal questionnaire when screening for health problems in the elderly. Gerontology, 25, 345-349.

\section{APPENDIX \\ COLOR VISION SCREENING INVENTORY*}

\section{Instructions}

This questionnaire deals with a number of common perceptually related situations. For each question you should select the response which best describes you and your behaviors. You can select from among the following response alternatives:

\section{Never (or almost never), Seldom, Occasionally, Frequently,}

Always (or almost always)

Simply place a check next to the letter which corresponds to the first letter of your choice.

1. Do you have difficulty discriminating between yellow and orange?

$\mathrm{N} \square \mathrm{S} \square \mathrm{O} \square \mathrm{F} \square \mathrm{A} \square$

2. Do you have difficulty discriminating between yellow and green?

$\mathrm{N} \square \mathrm{S} \square \mathrm{O} \square \mathrm{F} \square \mathrm{A} \square$

3. Do you have difficulty discriminating between gray and blue-green?

$\mathrm{N} \square \mathrm{S} \square \mathrm{O} \square \mathrm{F} \square \mathrm{A} \square$

4. Do you have difficulty discriminating between red and brown?

5. Do you have difficulty discriminating between green and brown?

$\mathrm{N} \square \mathrm{S} \square \mathrm{O} \square \mathrm{F} \square \mathrm{A} \square$

6. Do you have difficulty discriminating between pale green and pale red?

$N \square S \square O \square F \square A$

7. Do you have difficulty discriminating between blue and purple?

$\mathrm{N} \square \mathrm{S} \square \mathrm{O} \square \mathrm{F} \square \mathrm{A} \square$

8. Do the color names that you use disagree with those that other people use?

$N \square S \square O \square F \square \square$

9. Are the colors of traffic lights difficult to distinguish?

$\mathbf{N} \square \mathrm{S} \square \mathrm{O} \square \mathrm{F} \square \mathrm{A} \square$

10. Do you tend to confuse colors?

\section{Scoring Instructions}

Responses are scored 1 for Never, 2 for Seldom, 3 for Occasionally, 4 for Frequently, and 5 for Always. The total score is simply the sum of the 10 responses. Diagnostic cutoff points may be selected from Table 2. (In previous testing, these questions were distributed as part of an inventory containing other visual questions.)

*The Color Vision Screening Inventory is copyrighted by SC Psychological Enterprises Ltd., and reprinted by permission. It may be reproduced for research purposes only. We would appreciate receipt of copies of any data collected using this instrument as we are trying to establish population norms to assist researchers in interpretation of data collected with the CSI.

(Manuscript received May 4, 1987;

revision accepted for publication July $31,1987$. 